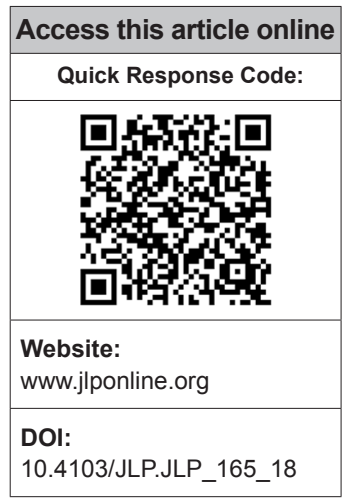

Department of Microbiology, All India Institute of Medical Sciences, Rishikesh, Uttarakhand, India

Address for correspondence: Dr. Mohit Bhatia, All India Institute of Medical Sciences, Rishikesh,

Uttarakhand, India E-mail: docmb1984@ gmail.com

Submission: 08-12-2018 Accepted: 13-02-2019

\title{
Salmonella osteomyelitis: A rare extraintestinal manifestation of an endemic pathogen
}

\author{
Ranjana Rohilla, Mohit Bhatia, Pratima Gupta, Arpana Singh, Ravi Shankar, \\ Balram Ji Omar
}

Abstract:

Salmonella enterica serovar Typhi and Paratyphi usually cause enteric fever in humans characterized by fever and gastrointestinal symptoms such as diarrhea. Bacteremia is a constant feature of enteric fever, and occasionally, dissemination of bacilli throughout the body results in the establishment of one or more localized foci of persisting infection. This happens especially in patients with preexisting conditions such as hemoglobinopathies, previous joint trauma, surgery, connective tissue diseases, lymphoma, diabetes, alcoholism, immunosuppressive state, and extremes of ages. Rarely, these extraintestinal Salmonella infection cases have also been reported in immunocompetent individuals without any obvious predisposing factors. Salmonella osteomyelitis, a rare sequelae of typhoid, may occur as a result of hematogenous seeding of the bone or joints due to a bacteremic episode. It may also spread from nearby areas of infection or penetrating trauma. Timely diagnosis and treatment of this condition are necessary because if left untreated, it can lead to permanent functional deficits later on. We hereby present a case series with brief review of literature on Salmonella osteomyelitis in immunocompetent patients without any known predisposing conditions over a period of 1 year (2017-2018) from a Tertiary Care Teaching Hospital in Uttarakhand, India.

Key words:

Enteric fever, immunocompetent, Salmonella osteomyelitis

\section{Introduction}

Tere he genus Salmonella consists of enteropathogenic Gram-negative bacilli comprising of two species namely Salmonella enterica and Salmonella bongori..$^{[1]}$ An estimated 11-20 million people get sick from typhoid and between 1, 28,000 and 1, 61,000 people die from it every year. ${ }^{[2,3]}$

It is an important public health problem in developing countries like India. Morbidity and mortality owing to these infections are increasing with the emergence of multidrug resistance. In addition, strains cause infections at aberrant sites, i.e., extraintestinal infections which cause increased morbidity.

This is an open access journal, and articles are distributed under the terms of the Creative Commons Attribution-NonCommercial-ShareAlike 4.0 License, which allows others to remix, tweak, and build upon the work non-commercially, as long as appropriate credit is given and the new creations are licensed under the identical terms.

For reprints contact: reprints@medknow.com
S. enterica serovar Typhi and Paratyphi usually cause enteric fever in humans characterized by fever and gastrointestinal symptoms such as diarrhea. Bacteremia is a constant feature of enteric fever, and occasionally, dissemination of bacilli throughout the body results in establishment of one or more localized foci of persisting infection. This happens especially in patients with preexisting conditions such as hemoglobinopathies, previous joint trauma, surgery, connective tissue diseases, lymphoma, diabetes, alcoholism, immunosuppressive state, and extremes of ages. ${ }^{[4]}$

Rarely, these extraintestinal Salmonella infection cases have also been reported in immunocompetent individuals without any obvious predisposing factors. ${ }^{[5]}$ Salmonella

How to cite this article: Rohilla R, Bhatia $M$, Gupta P, Singh A, Shankar R, Omar BJ. Salmonella osteomyelitis: A rare extraintestinal manifestation of an endemic pathogen. J Lab Physicians 2019;11:164-70. 
osteomyelitis, a rare sequelae of typhoid, may occur as a result of hematogenous seeding of the bone or joints due to a bacteremic episode. It may also spread from nearby areas of infection or penetrating trauma. Symptoms may include pain in a specific bone with overlying redness, fever, and weakness. Diagnosing and treating on time is necessary because if left untreated, it can lead to permanent functional deficits later on.

We hereby present a case series on Salmonella osteomyelitis with brief review of literature in immunocompetent patients without any known predisposing conditions over a period of 1 year (2017-2018) from a tertiary care teaching hospital in Uttarakhand, India.

\section{Case Reports}

\section{Case 1}

An 80-year-old male patient came to the orthopedics outpatient department with chief complaints of acute pain and restriction of movements in the right knee for 5 days. The patient did not give any history of fever over the preceding months. There was no history of trauma, prior surgery, abdominal discomfort, or any illness suggestive of enteric fever. Local examination of the right knee showed red, swollen joint with severe tenderness and the movements were painfully restricted. The laboratory findings were hemoglobin $12.8 \mathrm{~g} / \mathrm{dl}$ and white blood cell count 12,180 cells/cu. mm with neutrophilic leukocytosis. Erythrocyte sedimentation rate (ESR) was $32 \mathrm{~mm} / \mathrm{h}$. X-ray right knee showed age-related early osteoarthritic changes as depicted in Figure 1. Pus was aspirated from the knee joint and was inoculated in brain heart infusion broth, nutrient agar, 5\% sheep blood agar, and MacConkey agar and incubated overnight aerobically. Gram-stained smear of pus showed plenty of pus cells and Gram-negative

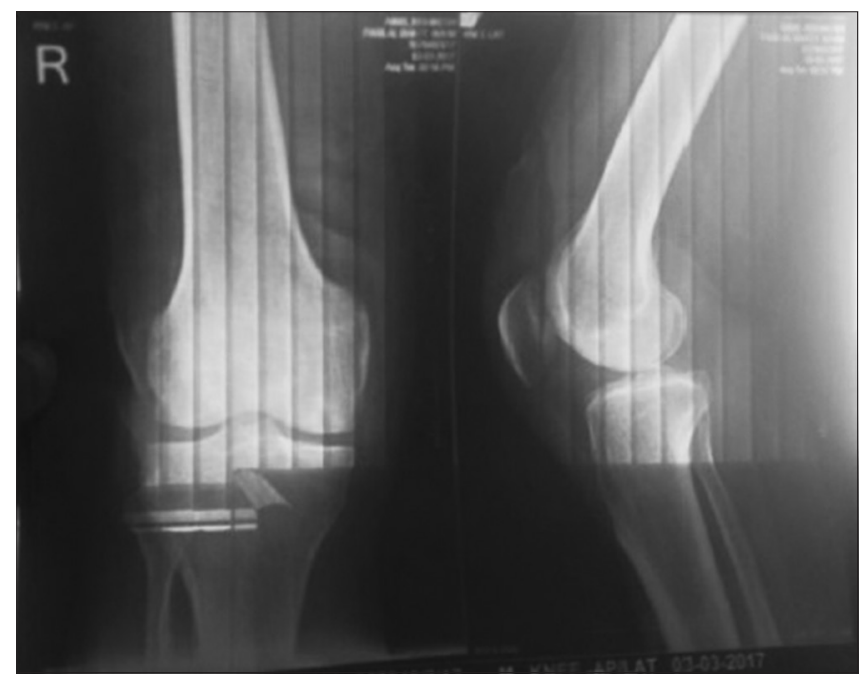

Figure 1: Anteroposterior and lateral views of $\mathrm{X}$-ray right knee showing age-related early osteoarthritic changes bacilli. Aerobic culture yielded S. enterica subspecies enterica serovar Typhi using conventional methods and serotyping (Difco, Becton and Dickinson, New Jersey, USA) ${ }^{[6]}$ Nonlactose-fermenting colonies obtained on MacConkey agar have been shown in Figure 2.

The isolate was found to be susceptible to ampicillin, chloramphenicol, ciprofloxacin, cefotaxime, ceftriaxone, cefepime, piperacillin-tazobactam, and amoxicillin-clavulanic acid by Kirby-Bauer disk diffusion method.

Blood culture, Widal test, and stool culture were simultaneously performed to search for foci of infection. Blood sample of this patient was subjected to aerobic culture by BACTEC 450 automated blood culture system (Difco, Becton and Dickinson, New Jersey, USA) and was found sterile. Both Widal test and stool culture were nonsignificant.

Treatment with ciprofloxacin $500 \mathrm{mg}$ twice daily was instituted for 3 weeks along with repeated aspirations. On follow-up, the patient was relatively asymptomatic. ${ }^{[7]}$

\section{Case 2}

A 17-year-old male patient presented with complaints of backache in the lumbar region for 1 month and reduced sensation in both lower limbs. On physical examination, the patient was afebrile with excessive paraspinal muscle spasm and lumbar lordosis. There was severe tenderness over L2-L5 region with restriction of movements. Neurological examination revealed sensory deficit below L4 level bilaterally, but there was neither bowel/bladder involvement nor saddle anesthesia. The total leucocyte count, ESR, and C-reactive protein were found to be 12,540 cells/cu. mm, $92.5 \mathrm{~mm} / \mathrm{h}$, and $14 \mathrm{mg} / \mathrm{L}$, respectively. Figure 3 shows $X$-ray spine which revealed paradisical erosions at the level of L3-L4 with the reduction of disc spaces. Magnetic

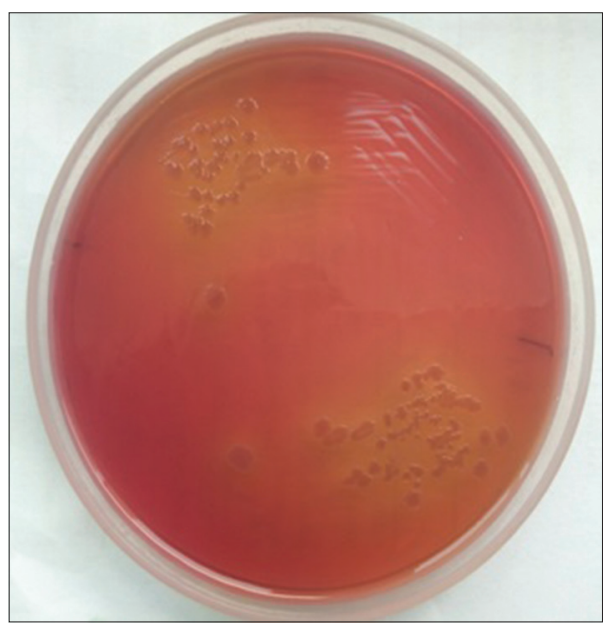

Figure 2: Nonlactose-fermenting colonies obtained on MacConkey agar 


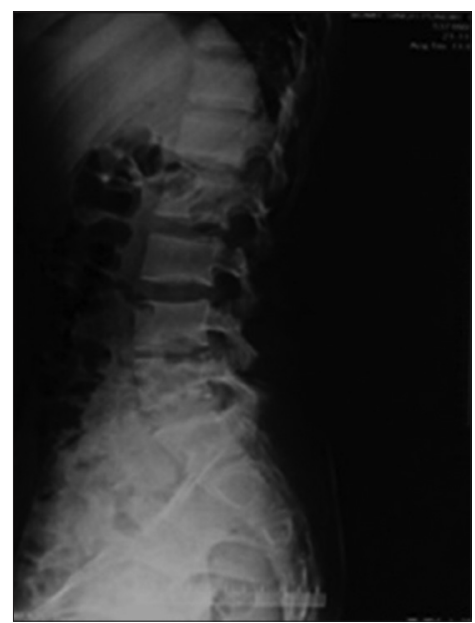

Figure 3: Preoperative $X$-ray lateral view showing reduction of disc spaces between L4 and L5 with irregularity of end plates

resonance imaging (MRI) of dorsolumbar spine showed spondylodiscitis at L2-L5 and epidural collection at L4-L5 level as depicted in Figure 4.

Correlating patient's history and radiological findings, a provisional diagnosis of Pott's spine was made, and antitubercular therapy (ATT) was started. On the $5^{\text {th }}$ day of admission, the patient gave a history of 2-3 episodes of passing loose stools with blood which subsided on its own. Keeping in mind the possibility of any pathology of gastrointestinal tract coexisting with the spinal condition, a detailed history was taken. On repeated history taking, it was revealed that the patient had fever and abdominal pain 3 months before developing back pain. He was prescribed antipyretics and analgesics which provided partial relief, but subsequently, he had loss of appetite and weight. Microscopic examination of stool sample did not reveal the presence of ova or cysts. Blood samples were collected from three different sites and subjected to aerobic culture using BACTEC-450-automated blood culture system (Becton and Dickinson, New Jersey, United States). S. enterica subspecies enterica serovar Typhi was isolated from all three samples. Identification was confirmed using conventional methods and serotyping using antisera (Difco, Becton and Dickinson, New Jersey, USA). Moreover, immunoglobulin M (IgM) immunochromatographic card test (Typhidot IgM, Enteroscreen - WB, Zephyr Biomedicals, Tulip Diagnostics Pvt. Ltd., Goa, India) was also positive.

The patient underwent open disc biopsy and decompressive laminotomy at L4-L5 region. Intraoperatively, aspirated pus was negative for acid-fast bacilli, but on aerobic culture, S. Typhi was isolated which was sensitive to aminoglycosides, cephalosporins (including ceftriaxone and cefuroxime), and $\beta$-lactam drugs by Kirby-Bauer disk diffusion method. Mycobacterial culture and Gene X-pert found no

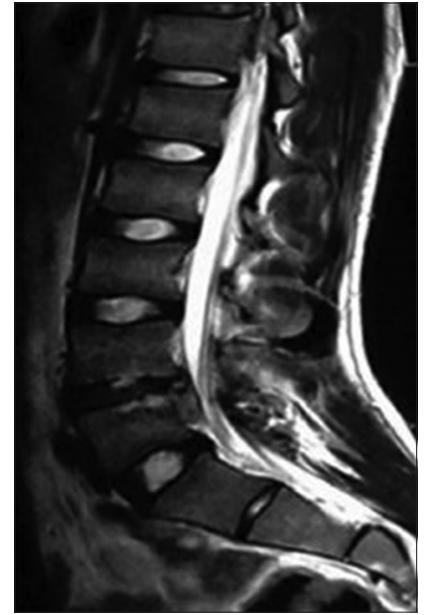

Figure 4: Preoperative T2-weighted magnetic resonance imaging of the patient showing spondylodiscitis at L4-L5 level with destruction of intervertebral disc, epidural collection, cord compression, and edema

evidence of tuberculosis. ATT was stopped immediately, and the patient was started on injection cefuroxime for 3 weeks on inpatient basis which was continued orally for a total of 6 weeks. The patient responded well to treatment and showed rapid symptomatic and neurological improvement. Follow-up after 6 months showed that the patient was asymptomatic with no neurological deficit. ${ }^{[8]}$

\section{Case 3}

A 24-year-old male was admitted with complaints of significant pain and swelling in the right thigh extending up to the knee for 3 months. He also had low-grade fever of 2 months' duration. His symptoms had worsened over the last 1 month as a result of which he found it exceedingly difficult to maintain an upright posture and walk. He also gave a history of diarrheal illness 3 months back, with complete recovery within 6-7 days without any medication. There was no history suggestive of hemoglobinopathy or any other underlying comorbidity. Conservative management by local health care practitioners using analgesics and physiotherapeutic measures over several weeks brought no relief to his current ailment.

At the time of presentation, the patient was conscious, oriented, and afebrile. Physical examination revealed tenderness and swelling over distal two-third of the right thigh over anterior aspect extending up to the knee joint. No scar, sinus, ulcer, or discharge was visible. On palpation, temperature was raised over the swelling, and distal pulses were palpable.

Completehaemogram revealed hemoglobin $11.3 \mathrm{~g} / \mathrm{dl}$, total leucocyte count of 21,720/cu. mm (neutrophils $-89.8 \%$ ), platelet count 2.92 lakhs/cu. mm, and ESR $65 \mathrm{~mm} / \mathrm{h}$. Liver and kidney function tests were within normal 
limits. X-ray showed ill-defined irregular intramedullary radiolucency over distal two-third of femur suggestive of osteolytic lesions with gross periosteal reaction and internal sclerotic bone fragments likely to be a sequestrum as depicted in Figure 5. Figure 6 shows MRI findings which revealed the presence of large intramedullary collection with sclerotic bone fragments at distal end of femur with marked synovial effusion and periarticular soft tissue edema suggestive of osteomyelitis.

Presumptive diagnosis of acute on chronic osteomyelitis of the right femur was made and cortical window with decompression surgery was planned. Cortical window was made with osteotome and blood-tinged pus was found in-between muscle planes and inside intramedullary cavity. Postoperatively, intravenous (IV) cefuroxime was given.

Pusfrom kneejointcavity, muscleplane, and intramedullary cavity showed growth of $S$. enterica subspecies enterica serovar Typhi identified by conventional methods and serotyping using antisera (Difco, Becton and Dickinson, New Jersey, USA). The isolate was sensitive to ampicillin, cotrimoxazole, ofloxacin, ciprofloxacin, ceftriaxone, and cefotaxime by Kirby-Bauer disk diffusion method. Aerobic blood culture was found to be sterile. The patient showed remarkable improvement postoperatively and was discharged while still on treatment with IV Ceftriaxone $2 \mathrm{~g}$ BD for 2 weeks.

\section{Discussion}

Enteric fever is caused by facultative intracellular organisms - Salmonella Typhi and Salmonella Paratyphi $\mathrm{A}$ and $\mathrm{B}$. It is more common among people traveling to or residing in developing countries like India where sanitation is poor and there is fecal contamination of food

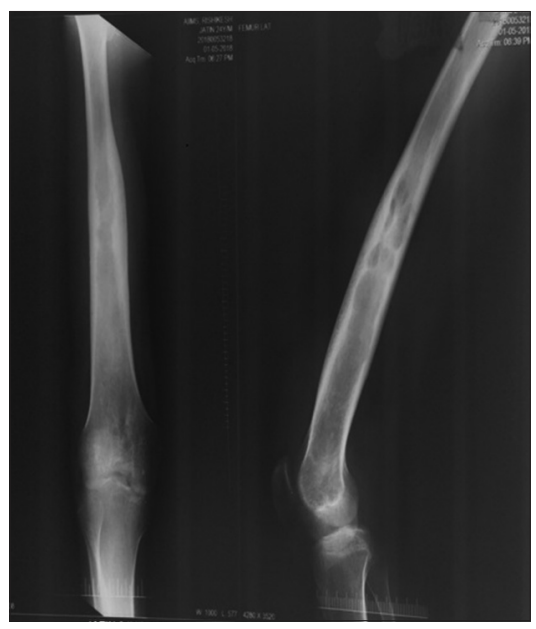

Figure 5: Anteroposterior and lateral views of X-ray showing ill-defined irregular intramedullary radiolucency over distal two-third of the right femur suggestive of osteolytic lesions and water. ${ }^{[9]}$ Enteric fever usually presents as acute febrile illness along with headache, diarrhea or constipation, relative bradycardia, splenomegaly, and leukopenia. Rarely, extraintestinal complications involving skeletal, cardiovascular, pulmonary, hepatobiliary, genitourinary, and central nervous systems are seen. ${ }^{[10]}$

Salmonella osteomyelitis is a rare presentation documented in hospitals, constituting $0.8 \%$ of all Salmonella infections and only $0.45 \%$ of all types of osteomyelitis occurring especially in sickle cell disease patients and caused usually by nontyphoidal Salmonellae. ${ }^{[11]}$ In sickle cell patients, capillary occlusion secondary to intravascular sickling may devitalize and infarct the gut, permitting Salmonella bloodstream invasion thereby increasing the chances of osteomyelitis. Complement system and impaired opsonization have also been suggested to play a role. ${ }^{[12,13]}$

Incidence of Salmonella osteomyelitis or arthritis is very rare in apparently healthy individuals with no predisposing factors. Cumulative records of Salmonella osteomyelitis cases in healthy adults (since 2010) from various parts of India have been enlisted in Table 1. Most of these cases have been reported from the southern part of the country. Salmonella osteomyelitis cases in healthy adults from other countries of the world (since 2010) have also been depicted in Table 2 .

At our center, 35 culture-proven cases of enteric fever and 3 cases of extraintestinal salmonellosis (osteomyelitis) caused by Salmonella Typhi were reported during the year 2017-2018, and in India, owing to its endemicity unlike other countries, Typhi is the main cause of Salmonella osteomyelitis.

First, these cases are unique owing to the rarity with which they occur. Second, three cases of Salmonella osteomyelitis from a tertiary care center in a year indicate

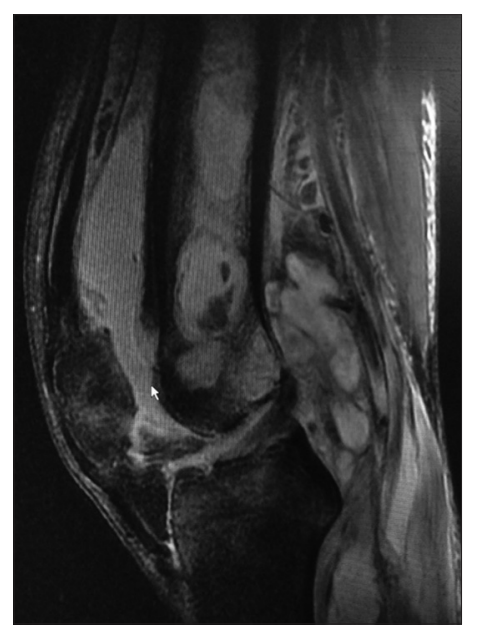

Figure 6: Magnetic resonance imaging showing large intramedullary collection with sclerotic bone fragments at the distal end of the right femur 
toward some indigenous risk factors predisposing to these extraintestinal manifestations in this region. The Salmonellae surviving in aberrant sites and persisting thereafter in vivo might be due to the insufficient treatment given for enteric fever. Inadequate dosage prescribed by quacks or other conventional treatment modalities may lead to the subsiding of symptoms but not complete cure (as was noted on careful history taking of these three cases), leading to persisting infection.

"Persisters" are defined as quiescent bacterial cells that survive lethal antibiotics or stresses but can regrow under appropriate conditions. These organisms are primarily responsible for latent infections, extraintestinal infections, and posttreatment relapse, posing significant challenges

Table 1: Cumulative records (since 2010) of Salmonella osteomyelitis cases in healthy adults from different parts of India ${ }^{[7,8,14-24]}$

\begin{tabular}{|c|c|c|}
\hline Zone & Site of involvement & References \\
\hline \multirow[t]{3}{*}{ North } & Dorsolumbar vertebra & Salmonella TyphiMohanty et al. \\
\hline & Knee joint & Salmonella TyphiGupta et al. \\
\hline & Hip joint & Salmonella TyphiGupta et al. \\
\hline West & Sacroiliac joint & Salmonella TyphiPhadke et al. \\
\hline Central & Thoracic spine vertebra & Salmonella TyphiGupta et al. \\
\hline \multirow[t]{8}{*}{ South } & Lumbar vertebrae & Salmonella TyphiBanerjee et al. \\
\hline & Thoracic spine & $\begin{array}{l}\text { Salmonella } \quad \text { Lakshmi et al. } \\
\text { enteritidis }\end{array}$ \\
\hline & $\begin{array}{l}5 \text { cases of osteomyelitis } \\
\text { of unspecified sites }\end{array}$ & s Salmonella TyphiSudhaharan et al. \\
\hline & Distal femur & Salmonella TyphiRanjith et al. \\
\hline & Rib & Salmonella TyphiMathuram A et al. \\
\hline & Hip joint & Salmonella TyphiShanthi et al. \\
\hline & Right acetabulum & \multirow{2}{*}{ Salmonella TyphiFaseela et al. } \\
\hline & Cranial vault & \\
\hline
\end{tabular}

for the treating physicians. Ying Zhang proposed a Yin-Yang model to describe the heterogeneous nature of persisters. A number of genes and pathways have been discovered which are as follows: toxin-antitoxin modules, stringent response, DNA repair or protection, phosphate metabolism, alternative energy production, efflux, antioxidative defense, and macromolecule degradation. Therefore, symptoms abate for some time with or without antibiotic treatment. Complications encountered at a later stage maybe due to the bacteria persisting inside body having adapted themselves to lesser-known niches. ${ }^{[40,41]}$

Salmonella osteomyelitis can be diagnosed by isolation of Salmonella species from the affected site. Simultaneous isolation of Salmonella spp. from blood and affected musculoskeletal site substantiates the diagnosis of disseminated Salmonella infection as seen in Case 2. Inability to isolate Salmonella spp. from blood samples in suspected cases of osteomyelitis as seen in Cases 1 and 3, respectively, can possibly be explained by the fact that inappropriate and inadequate antibiotic treatment usually results in chronic persistent infections which may exhibit the phenomenon of intermittent bacteremia. This issue can to some extent be addressed by obtaining adequate volumes of repeat blood samples from different sites at regular time intervals. Negative stool culture result in Case 1 can be explained by intermittent shedding of the bacterium in stool samples of infected patients. Insignificant Widal test results in the same case which could possibly be due to early and inadvertent administration of antibiotics (although a clear-cut past medical and treatment history was not available) and waning humoral immune response.

Table 2: Cumulative records of Salmonella osteomyelitis cases in healthy adults from other countries of world since 2010[25-39]

\begin{tabular}{llll}
\hline Country & Site of involvement & Isolate & References \\
\hline Greece & & Developed countrieCs & Papaioannou et al. \\
United States & Thoracic spine & S. enteritidis & Zaid et al. \\
& Spondylitis & Nontyphoidal Salmonella (not specified) & Shrestha et al. \\
Singapore & Thoracic vertebra & Salmonella Typhi & Khoo et al. \\
Japan & Vertebral osteomyelitis & Salmonella Typhi & Oki et al. \\
& Vertebra & S. enteritidis & Tonogai et al. \\
United Kingdom & Distal radius & Salmonella spp. (not specified) & Scarci et al. \\
& Rib & & Le Doare et al. \\
Korea & Sacroiliac joint & S. Typhimurium & Kim et al. \\
Spain & Femoral diaphysis & Salmonella Mbandaka & Martínez-Serrano et al. \\
Germany & Tibial diaphysis & Nontyphoidal Salmonella (not specified) & Salem et al. \\
Turkey & Knee joint & Salmonella spp. (not specified) & Uygur et al. \\
\hline & Knee joint & S. enteritidis & Alhazzazi et al. \\
\hline Saudi Arab & & S. enteritidis & Ayeni et al. \\
Kenya & Hip arthritis & Developing countries & Angela et al. \\
China & Distal radius (left forearm) & Salmonella (not specified) & Salmonella Paratyphi A
\end{tabular}


In all the three cases we are reporting, Salmonella osteomyelitis was not initially suspected, and no identifiable risk factors were noted. Since this entity is clinically as well as radiographically similar to osteomyelitis caused by other pyogenic agents, it is essential to diagnose and treat such cases accordingly. Bone tumors such as Ewing's sarcoma and fibrous dysplasia are also important differential diagnosis of pyogenic osteomyelitis and they may have very similar clinical presentations.

To conclude, clinicians should keep the possibility of Salmonella as an etiological agent in patients with osteomyelitis, especially in those giving a history of preceding fever with or without gastrointestinal manifestations even in the absence of underlying comorbidities like hemoglobinopathies. Furthermore, healthcare practitioners should ensure that each enteric fever patient receives appropriate antibiotics in the correct dose and for the right duration. In addition, antibiotic susceptibility test results of Salmonella isolates should be used to guide proper therapy. Restriction of indiscriminate use of antibiotics in these cases would further prevent emergence and spread of multidrug resistance.

\section{Declaration of patient consent}

The authors certify that they have obtained all appropriate patient consent forms. In the form the patient(s) has/ have given his/her/their consent for his/her/their images and other clinical information to be reported in the journal. The patients understand that their names and initials will not be published and due efforts will be made to conceal their identity, but anonymity cannot be guaranteed.

\section{Financial support and sponsorship \\ Nil.}

\section{Conflicts of interest}

There are no conflicts of interest.

\section{References}

1. Popoff MY, Minor LL. WHO collaborating centre for reference and research on Salmonella. Antigenic Formulas of the Salmonella Serovars. $7^{\text {th }}$ Revision. Paris, France: Institut Pasteur; 1997.

2. Giannella RA. Salmonella. In: Baron S, editor. Medical Microbiology. $4^{\text {th }}$ ed., Ch 21. Galveston (TX); Univ of Texas Medical Branch: 1996.

3. World Health Organization. Typhoid. World Health Organization; 2018. Available from: http://www.who.int/immunization/ diseases/typhoid/en/. [Last updated 2018 Sep 11; Last accessed 2018 Sep 20].

4. KaisthaN,Gupta V,SidhuS, ChanderJ.Salmonella-salmonellosis-rare presentations of a common pathogen. Asian Pac J Trop Med 2011;4:417-20.

5. Arora A, Singh S, Aggarwal A, Aggarwal PK. Salmonella osteomyelitis in an otherwise healthy adult male-successful management with conservative treatment: A case report. J Orthop Surg (Hong Kong) 2003;11:217-20.

6. Old DC. Salmonella. In: Collee JG, Fraser, AG, Marmion BP, Simmons A, editors. Mackie and Mccartney Practical Medical Microbiology. $14^{\text {th }}$ ed. New Delhi: Elsevier; 2014. p. 385-402.

7. Gupta P, Kaistha N, Omar BJ, Gupta P, Singh V, Mohanty A. Salmonella typhi: A cause of septic arthritis knee: A rare entity. Int J Biomed Res 2017;8:242-4.

8. Mohanty A, Pandita N, Kandwal P, Omar BJ, Gupta P, Gupta P. Salmonella osteomyelitis of the lumbar spine: An unusual presentation in an immunocompetent male. Asian J Pharm Clin Res 2018;11:1-2.

9. Kothari A, Pruthi A, Chugh TD. The burden of enteric fever. J Infect Dev Ctries 2008;2:253-9.

10. Huang DB, DuPont HL. Problem pathogens: Extra-intestinal complications of Salmonella enterica serotype typhi infection. Lancet Infect Dis 2005;5:341-8.

11. McAnearney S, McCall D. Salmonella osteomyelitis. Ulster Med J 2015;84:171-2.

12. Sanchez AA, Mazurek MT, Clapper MF. Salmonella osteomyelitis presenting as fibrous dysplasia. A case report. Clin Orthop Relat Res 1996;330:185-9.

13. Anand AJ, Glatt AE. Salmonella osteomyelitis and arthritis in sickle cell disease. Semin Arthritis Rheum 1994;24:211-21.

14. Gupta V, Priyadarshi A, Mehra N, Yadav TP, Dewan V. Salmonella typhi causing hip arthritis with dislocation. JIACM 2014;15:141-2.

15. Phadke PS, Gandhi AR, More SA, Joshirao RP. Salmonella pyomyositis with concurrent sacroiliac osteomyelitis presenting as piriformis syndrome: A rare case. J Postgrad Med 2017;63:44-6.

16. Gupta R, Awasthi R, Pauranik A. Salmonella vertebral osteomyelitis of thoracic spine. J Spine Surg 2014;1:135-7.

17. Banerjee B, Madiyal M, Madhava PK, Agarwal M, Mukhopadhyay C. Typhoid spondylodiscitis mimicking tuberculosis in a teenage girl. J Infect Public Health 2018;11:136-7.

18. Lakshmi K, Santhanam R. Thoracic spinal osteomyelitis due to Salmonella enteritidis in an immunocompetent mimicking tuberculosis. J Neurosci Rural Pract 2016;7:317-9.

19. Sudhaharan S, Padmaja K, Solanki R, Lakshmi V, Umabala P, Aparna B, et al. Extra-intestinal salmonellosis in a tertiary care center in South India. J Infect Dev Ctries 2014;8:831-7.

20. Ranjith TC, Bhaskaran VK. Salmonella typhi osteomyelitis in healthy young adult. Kerala J Orthop 2013;26:113-5.

21. Mathuram A, Rijn RV, Varghese GM. Salmonella typhi rib osteomyelitis with abscess mimicking a 'cold abscess'. J Glob Infect Dis 2013;5:80-1.

22. Shanthi M, Sekar U, Sridharan KS. Septic arthritis of hip caused by Salmonella typhi: A case report. Case Rep Infect Dis 2012;2012:464527.

23. Faseela TS, Malli CS, Balakrishna AK, Gomes L, Nayak N. Salmonella typhi septic arthritis of the hip - A case report. J Clin Diagn Res 2010;4:2308-10.

24. Bhooshan P, Shivaprakasha S, Dinesh KR, Kiran M, Karim P. Chronic subdural empyema and cranial vault osteomyelitis due to Salmonella paratyphi A. Indian J Med Microbiol 2010;28:60-2.

25. Papaioannou I, Baikousis A, Korovessis P. Multi-foci Salmonella enteritis osteomyelitis of thoracic spine with pleural effusion and fatal outcome. A unique case presentation and review of the literature. J Orthop Case Rep 2017;7:69-74.

26. Zaid B, Jebaje AL, Zhao A, Samannodi M, Al-Sofiani M, Hocko M. Salmonella spondylitis in an immunocompetent non-sickle cell patient. Int J Case Rep 2017;8:187-90.

27. Shrestha P, Mohan S, Roy S. Bug on the back: Vertebral osteomyelitis secondary to fluoroquinolone resistant Salmonella typhi in an immunocompetent patient. BMJ Case Rep 2015;2015. pii: bcr2015212503.

28. Khoo HW, Chua YY, Chen JL. Salmonella typhi vertebral 
osteomyelitis and epidural abscess. Case Rep Orthop 2016;2016:6798157.

29. Oki M, Ueda A, Tsuda A, Yanagi H, Ozawa H, Takagi A, et al. Salmonella enterica serotype enteritidis vertebral osteomyelitis and epidural abscess complicated with meningitis. Tokai J Exp Clin Med 2016;41:169-71.

30. Tonogai I, Hamada Y, Hibino N, Sato R, Henmi T, Sairyo K, et al. Salmonella osteomyelitis of the distal radius in a healthy young adult patient: Report of a rare case and literature review. J Med Invest 2015;62:97-9.

31. Scarci M, Attia R, Phipps KH. Look what's eroding through the chest wall? Salmonella osteomyelitis of the ribs in an immunocompetent adult not associated with sickle cell disease. Ann R Coll Surg Engl 2010; 92:59-61.

32. Le Doare K, Brooker E, Ladhani S. Travel-associated Salmonella mbandaka sacroiliac osteomyelitis in a healthy adolescent. Case Rep Infect Dis 2013;2013:543147.

33. Kim BK, Dan J, Lee YS, Kim SH, Cha YS. Salmonella osteomyelitis of the femoral diaphysis in a healthy individual. Am J Orthop (Belle Mead NJ) 2014;43:E237-9.

34. Martínez-Serrano M, Arranz-Solana C, Martínez-Alfaro E, Crespo-Sánchez MD. Right knee pain, swelling and fever in a young immunocompetent male. Enferm Infecc Microbiol Clin 2014;32:396-7.

35. Salem KH. Salmonella osteomyelitis: A rare differential diagnosis in osteolytic lesions around the knee. J Infect Prev 2014;7:66-9.

36. Uygur E, Reddy K, Ozkan FÜ, Söylemez S, Aydin O, Senol S. Salmonella enteridis septic arthritis: A report of two cases. Case Rep Infect Dis 2013;2012:3. Available from: https://www. ncbi.nlm.nih.gov/pmc/articles/PMC3819818/pdf/CRIM. ID2013-642805.pdf. [Last accessed on 2018 Sep 20].

37. Alhazzazi KM, Alsheikh K, Konbaz F, AlMugren TS. Salmonella species causing hip septic arthritis. Int J Community Med Public Health 2018;5:3146-8.

38. Ayeni IV, Calver G. Ciprofloxacin resistant osteomyelitis following typhoid fever. BMJ Case Rep 2012;2012. pii: bcr0320126107.

39. Angela HW, Tong CS, Lam CW, Hung YC, Cheung WW. Salmonella osteomyelitis of the pelvis in healthy adults: A case report. J Orthop Trauma Rehabil 2011;15:27-8.

40. Zhang Y. Persisters, persistent infections and the yin-yang model. Emerg Microbes Infect 2014;2012:6. Available from: https:/ / www. nature.com/articles/emi20143.pdf. [Last accessed on 2018 Sep 20].

41. Lew DP, Waldvogel FA. Osteomyelitis. Lancet 2004;364:369-79. 